\title{
Thermodynamics of Perfect Elastic Fluids
}

\author{
B. Bernstein, E. A. Kearsley, and L. J. Zapas
}

(May 20, 1964)

\begin{abstract}
A simple non-equilibrium thermodynamics is developed and a particular example is studied. The theory is formulated to describe a viscoelastic fluid, capable of finite deformation, which need not be locally in or near a state of thermodynamic equilibrium. This fluid may support shear stresses only when away from local thermodynamic equilibrium. A notion of time-temperature superposition is contained in the formulation of the constitutive equations. Conservation of energy is obeyed and the second law of thermodynamics is satisfied as a consequence of simple requirements on the constitutive relations. In an adiabatic isochoric motion the temperature increases when work is done on the material and decreases when the material does work. For given volume and temperature, entropy decreases when the material is deformed from equilibrium. It is shown in what general way viscosity depends upon temperature. For infinitesimal strain, the special form of the stress-strain relations are derived in order to determine how temperature and time-temperature superposition enter in this case.
\end{abstract}

\section{Introduction}

In 1963 we established that a fluid theory gives the correct stress relaxation response for a large variety of materials, some of which, such as vulcanized butyl rubber, are usually treated as solids [1]. ${ }^{1}$ We started then to investigate whether or not it would be fruitful to regard all matter as fluid. Subsequent experimental work [2] has indicated not only that the fluid point of view can be productive in a practical sense, but that the incompressible elastic fluid theory of our 1963 article is also very promising. It is thus a natural step, at this juncture, to endeavor to extend our theoretical understanding by attempting to create a thermodynamic theory, while deliberately adhering to the fluid point of view and bearing in mind that the incompressible elastic fluid should represent an isothermal limit. We believe that the fluid notion is the key to a simple thermodynamics just as it was the key to the simple isothermal mechanical theory.

We cannot reconcile the notion of a fluid with the notion of equilibrium with shear stresses present. Consequently from this viewpoint, even materials usually considered to be solid, such as vulcanized rubber, cannot be considered in equilibrium while supporting shear. If we are to describe shear, we are therefore left with no alternative other than to write a non-equilibrium thermodynamics.

We offer a caveat. Because the non-equilibrium concept is essential to make the fluid point of view hold together, the reader who reflects on solidlike behavior of our fluid must not forget that we have given up thinking of a sheared state in terms of classical equilibrium thermodynamics. Otherwise he will arrive at paradoxes.

' Figures in brackets indicate the literature references on page 112.
The problem of how to generalize equilibrium thermodynamics to include non-equilibrium relations is resolved by adjoining to the usual state variables a quantity which has dimensions of specific entropy and which depends upon the past history of the material. ${ }^{2}$ This quantity is the seat of irreversibility. With it we generate a statement of the second law which is meaningful for non-equilibrium. With it we also arrive at a thermodynamic compressible fluid theory which allows solidlike behavior, and which is consistent with the incompressible elastic fluid theory cited above.

The basic definitions, assumptions, and equations which constitute the thermodynamic theory of perfect elastic fluids are given in section 2. Central to the understanding of these is the idea of local thermodynamic equilibrium or non-equilibrium and the relations which hold in each case. Section 3 is devoted to the manner in which the notion of time-temperature superposition enters the theory. It is suggested that the reader who is anxious to get on to the questions of energy conservation and dissipation may skip or skim through section 3 in his first reading. In section 4 we write down the form which the equation of conservation of energy takes in the context of a perfect elastic fluid. Our statement of fading memory, together with nonnegative heat conductivity, is shown to imply consistency with the second law of thermodynamics in section 5 . Also for given specific volume and temperature, the entropy will be less in local non-equilibrium than in local equilibrium. In section 6 we show that positive heat capacity at constant deformation implies that temperature is rising when work is being done on the material and falling when work is being done by the material isochorically and adiabatically. Upper bounds are obtained on the amount of work available by allowing a material to recover either adiabatically 
or isothermally. In section 7 we derive a relation between temperature and viscosity which has been observed experimentally. And in the final section, section 8 , we obtain the stress-strain relations for infinitesimal strain.

\section{The Caloric Equation of State}

In the theory of inviscid gases, the caloric equation of state relates specific internal energy $\epsilon$ to specific volume $v$ and specific entropy $s$ through an expression of the form

$$
\epsilon=f(v, s),
$$

A stipulation of the form of the function $f$ suffices to characterize the gas. The functions relating local pressure $p$ and local absolute temperature $T$ to specific volume and specific entropy are given by

$$
\begin{gathered}
p=-f_{v}(v, s) \equiv-\frac{\partial f}{\partial v} \\
T=f_{s}(v, s) \equiv \frac{\partial f}{\partial s},
\end{gathered}
$$

so that one has the familiar relation

$$
d \epsilon=T d s-p d v \text {. }
$$

Although a given volume of gas need not always be in equilibrium because quantities such as temperature or pressure vary from point to point, yet at each point local thermodynamic equilibrium is assumed always to hold in the sense that (2.1), (2.2), and (2.3) hold pointwise. When we turn to viscoelastic behavior, we take the point of view that such behavior cannot be explained by local thermodynamic equilibrium, and that a fluid in thermodynamic equilibrium cannot support shear stress. Thus we set ourselves the task of creating a theory which deals with fluids which are not in local thermodynamic equilibrium. We do this with caution, using equilibrium thermodynamic theory (theory of gases) as a basic guide, and departing conceptually from this theory in as little measure as we feel will allow us to explain observed viscoelastic phenomena. The theoretical material thus described will be called a perfect elastic fluid.

As will be made clear below, we incorporate into our theory the following notions

$1^{\circ}$ There exists locally a specific internal energy $\epsilon$ and a specific entropy $s$.

$2^{\circ}$ There exists locally a non-negative quantity $\Sigma$ having dimensions of entropy per unit mass, which vanishes at thermodynamic equilibrium. Indeed, local equilibrium at a point prevails if and only if $\Sigma=0$ at that point.

$3^{\circ}$ In equilibrium a caloric equation of state of the form (2.1) holds. Whether or not local equilibrium

2 In 1950, P. W. Bridgman published a very perceptive article [3] in which he speculated on some of the properties which a non-equilibrium theory of this type would have to exhibit. We have here a concrete example of such a theory which meets Bridgman's requirements. prevails, the caloric equation of state reads

$$
\epsilon=f(v, s+\Sigma) \text {. }
$$

$4^{\circ}$ There is a local absolute temperature $T$ which satisfies

$$
T=f_{s}(v, s+\Sigma) .
$$

$5^{\circ}$ The stress is given by a hydrostatic pressure $-p \delta_{i j}$ plus terms that vanish at equilibrium where

$$
p=-f_{v}(v, s+\Sigma) .
$$

In developing our set of notions into a theory, we have the task of relating $\Sigma$ and stress to other quantities. $^{3} \quad$ To this end, we now discuss some kinematics.

We use Cartesian tensor notation, letting the point $\mathbf{x}$ be given by Cartesian coordinates $x_{i}, i=1,2,3$. For a given motion, the position of a particle $\mathbf{X}$ at time $t, \mathbf{x}(t)$, can be expressed in terms of its position at time $\tau, \mathbf{x}(\tau)$, by a function

$$
x_{i}(t)=x_{i}[\mathbf{x}(\tau), t, \tau],
$$

from which we may define the relative deformation gradients

$$
x_{i k}(t, \tau) \equiv \frac{\partial x_{i}(t)}{\partial x_{k}(\tau)} .
$$

By a configuration, we simply mean an assignment of position $x_{i}$ to each particle $\mathbf{X}$.

The Cauchy-Green relative strain tensor $C_{i j}(t, \tau)$ is defined by

$$
C_{i j}(t, \tau)=x_{k i}(t, \tau) x_{k j}(t, \tau),
$$

and the Green-St. Venant relative strain tensor is

$$
E_{i j}(t, \tau)=\frac{1}{2}\left[C_{i j}(t, \tau)-\delta_{i j}\right] .
$$

For the matrix of $E_{k l}(t, \tau)$ and $C_{k l}(t, \tau)$ we write $\mathbf{E}(t, \tau)$ and $\mathbf{C}(t, \tau)$ respectively.

We return to the question of specifying $\Sigma$ and stress away from local thermodynamic equilibrium, and proceed by assuming the existence of a function

$$
S[\mathbf{E}(\xi, \tau), t]
$$

which is defined for all $t \geqslant 0$ and depends on a strain $\mathbf{E}(\xi, \tau)$ only through

$$
\begin{aligned}
& I_{1}(\xi, \tau)=\lambda_{1}^{2}+\lambda_{2}^{2}+\lambda_{3}^{2} \\
& I_{2}(\xi, \tau)=\lambda_{1}^{2} \lambda_{2}^{2}+\lambda_{2}^{2} \lambda_{3}^{2}+\lambda_{3}^{2} \lambda_{1}^{2} \\
& I_{3}(\xi, \tau)=\lambda_{1}^{2} \lambda_{2}^{2} \lambda_{3}^{2}
\end{aligned}
$$

${ }^{3}$ The incompressible elastic fluid [1] provided a key as to how to write the expression for stress. 
where $\lambda_{i}^{2}=\lambda_{i}^{2}(\xi, \tau), i=1,2,3$, are the principal values of $\mathbf{C}(\xi, \tau)$.

We write

$$
\begin{aligned}
S_{k l}[\mathbf{E}(\xi, \tau), t] & \equiv \frac{\partial S}{\partial E_{k l}} \\
S_{t}[\mathbf{E}(\xi, \tau), t] & \equiv \frac{\partial S}{\partial t},
\end{aligned}
$$

these notations applying no matter what name is given to the arguments of $S$.

Furthermore, we shall assume the existence of a material property $b_{T}>0$, which scales time intervals in a manner to be made more precise in section 3 below. The quantity $b_{T}$ depends on absolute temperature $T$ and has the value unity at a given reference temperature $T_{0}$. We now assert that the value of $\Sigma$ at present time $t$ depends upon the past history of deformation and temperature through ${ }^{4}$

$$
\Sigma(t)=\int_{-\infty}^{t} S\left[\mathbf{E}(t, \tau), \int_{\tau}^{t} b_{T}(\xi) d \xi\right] b_{T}(\tau) d \tau,
$$

where $b_{T}(\tau)$ means the value of $b_{T}$ at time $\tau$. Further we specify that stress $\sigma_{i j}$ at time $t$ satisfies ${ }^{5}$

$$
\sigma_{i j}=-p \delta_{i j}
$$

$+\rho T \int_{-\infty}^{t} x_{i k}(t, \tau) x_{j l}(t, \tau) S_{k l}\left[\mathbf{E}(t, \tau), \int_{\tau}^{t} b_{T}(\xi) d \xi\right] b_{T}(\tau) d \tau$

where $\rho=v^{-1}$ is density, $S_{k l}$ is defined by (2.11) and $p$ by (2.6).

(In (2.14), as subsequently, we do not mention explicitly that quantities such as $\sigma_{i j}, \rho, \Sigma$, and $T$ are evaluated at present time $t$ when the context makes this clear.)

We note that $s+\Sigma$ may be eliminated between (2.5) and (2.6) to express $p$ as a function of specific volume and local temperature, say

$$
p=g(v, T),
$$

whence (2.14) becomes

$\sigma_{i j}=-g(v, T) \delta_{i j}$

$$
+\rho T \int_{-\infty}^{t} x_{i k}(t, \tau) x_{j l}(t, \tau) S_{k l}\left[\mathbf{E}(t, \tau), \int_{\tau}^{t} b_{T}(\xi) d \xi\right] b_{T}(\tau) d \tau .
$$

The expression (2.14) bears a resemblance to the constitutive equation of an incompressible elastic fluid given by Bernstein, Kearsley, and Zapas, which is a corresponding isothermal incompressible theory.

${ }^{4}$ If in further discussions it should prove desirable to have a name for $\Sigma$, we suggest calling it entaxy.

${ }^{5}$ In equation (3.6) of reference [1], the potential $U$ of the isothermal theory of the in compressible fluid replaces $\rho T S$ in eq (2.14) of the present article.
Indeed, for constant temperature

$$
\int_{\tau}^{t} b_{T}(\xi) d \xi=(t-\tau) b_{T}
$$

in which case the stress is given by (2.14) as if each configuration that the material assumed in the past contributed to the stress as the preferred configuration of an elastic material, each contribution being weighted according to the time elapsed, added up, and modified by the addition of an appropriate hydrostatic pressure.

Since the role that $S$ plays is analogous to that of an elastic strain energy, it is reasonable to assume that

$$
S[\mathbf{E}(t, \tau), \xi] \geqslant 0
$$

for all $\xi \geqslant 0$ and all values of $\mathbf{E}(t, \tau)$ for which $S$ is defined, and that

$$
S[\mathbf{0}, \xi]=S[\mathbf{E}(t, t), \xi]=0
$$

for all $\xi \geqslant 0$, where $\boldsymbol{0}$ is the zero matrix.

From (2.17), it is clear that whenever $S$ vanishes it assumes a minimum value, whence

$$
S_{k l}[\mathbf{E}, \xi]=0 \text { whenever } S[\mathbf{E}, \xi]=0 .
$$

In particular, (2.18) and (2.19) yield

$$
S_{k l}[\mathbf{0}, \xi]=0
$$

for all $\xi \geqslant 0$.

Our theory has been set up to be consistent with $1^{\circ}, 2^{\circ}, 3^{\circ}$, and $4^{\circ}$. To show that it is also consistent with $5^{\circ}$, we have but to establish that the integral terms in (2.14) vanish when local thermodynamic equilibrium prevails, i.e., when $\Sigma=0$. But if $\Sigma=0$, we see from $(2.13),(2.17)$, and $b_{T}>0$, that

$$
S\left[\mathbf{E}(t, \tau), \int_{\tau}^{t} b_{T}(\xi) d \xi\right]
$$

vanishes for all $\tau \leqslant t$. Thus, (2.19) assures us that $S_{k l}$ also vanishes for all $\tau \leqslant t$ and hence, from (2.14), $\sigma_{i j}=-p \delta_{i j}$, which completes our argument that $5^{\circ}$ holds.

In addition to (2.17) and (2.18), we assume fading memory in the form

$$
S_{t} \leqslant 0 .
$$

For an interpretation of the motivation for this assumption, let us consider the case in which temperature, and hence $b_{T}$, is constant, so that

$$
\int_{\tau}^{t} b_{T}(\xi) d \xi=(t-\tau) b_{T}
$$

is proportional to the time interval between $t$ and $\tau$. Consider, then, a given value of relative strain $\mathbf{E}$, and consider the contribution to the internal energy which arises if the relative strain computed from the configurations at present time $t$ and past time $\tau$ takes this given value. Then, in view of (2.4) and (2.13), 
(2.21) says that this contribution cannot increase for larger values of the time interval $t-\tau$.

If up to and including present time the material has been motionless, or even just undeformed, i.e., $\mathbf{E}(t, \tau)=\mathbf{0}$ for $\tau \leqslant t$, then (2.13) and (2.18) imply that $\Sigma$ vanishes and thus local thermodynamic equilibrium prevails. But, furthermore, we can show that if our material is kept at constant deformation and at constant temperature from some time $\mathrm{t}_{1}$ onward, it will tend toward local thermodynamic equilibrium, i.e. If $\mathbf{E}(\mathrm{t}, \tau)=\mathbf{0}$ and $\mathrm{T}(\mathrm{t})=\mathrm{T}\left(\mathrm{t}_{1}\right)$ for all $\mathrm{t}, \tau \geqslant \mathrm{t}_{1}$, then

$$
\lim _{\mathrm{t} \rightarrow \infty} \Sigma(\mathrm{t})=0 \text {, }
$$

where $\Sigma(\mathrm{t})$ decreases monotonically when $\mathrm{t}>\mathrm{t}_{1}$.

Appendix A is devoted to the proof of this statement. Example: For an ideal gas

$$
\epsilon=D v^{(1-\gamma)} \exp \frac{s}{C_{V}},
$$

where $D>0, \gamma>1$ and $C_{V}>0$ are constants. Generalizing this equation slightly to allow a different dependence of $\epsilon$ on volume, we may consider

$$
\epsilon=\Upsilon(v) \exp \frac{s}{C_{V}},
$$

where $Y(v)$ is some given function of $v$. Starting with (2.22) in place of (2.1), we may form an elastic fluid for which (2.4) becomes

$$
\epsilon=\Upsilon(v) \exp \frac{1}{C_{V}}(s+\Sigma),
$$

where $\Sigma$ is given by an expression of the form (2.13). Then from (2.23) and (2.5) we obtain

$$
\epsilon=C_{V} T
$$

Also (2.15) becomes

$$
p=-[\ln Y(v)]^{\prime} C_{V} T,
$$

whence (2.16) reads

$$
\begin{aligned}
\sigma_{i j} & =[\ln Y(v)]^{\prime} C_{V} T \delta_{i j} \\
& +\rho T \int_{-\infty}^{t} x_{i k}(t, \tau) x_{j l}(t, \tau) S_{k l}\left[\mathbf{E}(t, \tau), \int_{\tau}^{t} b_{T}(\xi) d \xi\right] b_{T}(\tau) d \tau
\end{aligned}
$$

A perfect elastic fluid for which (2.23) holds may be called an ideal elastic fluid.

\section{Time-Temperature Superposition}

The idea of time-temperature superposition which we introduce is based on the notion of a clock belonging to each particle of the material. Our assumption is that the material reads its own clock, which is temperature sensitive and runs at a rate $b_{T}$ as fast as a standard or laboratory clock, which is not temperature sensitive.

If at time $t=0$, the temperature sensitive material clock reads $\theta_{0}$, then at time $t$ it reads $\theta(t)$ given by

$$
\theta(t)=\int_{0}^{t} b_{T}(\xi) d \xi+\theta_{0}
$$

At a constant temperature $T$, the material clock will advance

$$
\theta(t)-\theta(\tau)=(t-\tau) b_{T}
$$

when the laboratory clock advances $t-\tau$. At reference temperature $T_{0}$ the material clock and the laboratory clock run at the same rate, so that $b_{T}=1$ and $\theta(t)-\theta(\tau)=t-\tau$.

Looking at (2.15) now, we see that $p$ does not depend explicitly on the past history of deformation and temperature, but only on the present value of temperature and specific volume. Using (2.14) we see then, that $\left[\sigma_{i j}+p \delta_{i j}\right] / \rho T$ depends entirely on the histories of the temperature and the strain relative to the present configuration. The concept of time-temperature superposition commonly used in linear viscoelasticity [4] can be stated in terms of these ideas as follows:

Suppose that $\mathbf{x}=\mathbf{f}(t, \mathbf{X})$ is a motion occurring at reference temperature $T_{0}$ with corresponding specific volume $v(t, \mathbf{X})$ and stress $\sigma_{i j}(t, \mathbf{X})$ (where $\mathbf{X}$ is a particle label). If this motion is replaced by $\mathbf{x}=\mathbf{f}\left(b_{T} t, \mathbf{X}\right)$ at constant temperature $T$, with corresponding specific volume $v(t, \mathbf{X})=v\left(b_{T} t, \mathbf{X}\right)$ and corresponding density $\hat{\rho}(t, \mathbf{X})=\rho\left(b_{T} t, \mathbf{X}\right)$, then the corresponding stress $\hat{\sigma}_{i j}(t, \mathbf{X})$ will satisfy

$$
\frac{\hat{\sigma}_{i j}(t, \mathbf{X})+\hat{p}(t, \mathbf{X}) \delta_{i j}}{\hat{\rho}(t, \mathbf{X}) T}=\frac{\sigma_{i j}\left(b_{T} t, \mathbf{X}\right)+p\left(b_{T} t, \mathbf{X}\right) \delta_{i j}}{\rho\left(b_{T} t, \mathbf{X}\right) T_{0}}
$$

where

$$
p(t, \mathbf{X})=g\left[v(t, \mathbf{X}), T_{0}\right]
$$

and

$$
\hat{p}(t, \mathbf{X})=g[\hat{v}(t, \mathbf{X}), T]
$$

It is a simple matter to generalize this statement to apply to situations in which the temperature varies with time. We replace each time increment $d \tau$ by $b_{T}(\tau) d \tau$ and restate the principle as follows: Let $\mathbf{x}=\mathbf{f}(\mathrm{t}, \mathbf{X})$ be a given motion occurring at reference temperature $\mathrm{T}_{0}$ and corresponding specific volume $v(\mathrm{t})$ with stress $\sigma_{i j}(\mathrm{t}, \mathbf{X})$. Suppose that $\hat{\mathbf{x}}=\mathbf{f}(\theta(\mathrm{t}), \mathbf{X})$, with corresponding specific volume $\hat{v}(\mathrm{t}, \mathbf{X})=v(\theta(\mathrm{t}), \mathbf{X})$ and corresponding density $\hat{\rho}(\mathrm{t}, \mathbf{X})=\rho(\theta(\mathrm{t}), \mathbf{X})$ is a motion occurring at (possibly varying) temperature $\mathrm{T}(\mathrm{t})$ with corresponding $\mathrm{b}_{\mathrm{T}}(\mathrm{t})$, so that $\theta(\mathrm{t})$ is given by (3.1). Let $\hat{\sigma}_{i j}(\mathrm{t}, \mathbf{X})$ be the stress corresponding to the second motion. Then

$$
\frac{\hat{\sigma}_{i j}(t, \mathbf{X})+\hat{p}(t, \mathbf{X}) \delta_{i j}}{\hat{\rho}(t, \mathbf{X}) T}=\frac{\sigma_{i j}(\theta(t), \mathbf{X})+p(\theta(t), \mathbf{X}) \delta_{i j}}{\rho(\theta(t), \mathbf{X}) T_{0}}
$$


where

$$
p(t, \mathbf{X})=g\left(v(t, \mathbf{X}), T_{0}\right)
$$

$\frac{\hat{\sigma}_{i j}(t)+\hat{p}(t)}{\hat{\rho}(t) T(t)}=\int_{-\infty}^{t} \pi_{i j}\left[x_{k l}(\theta(t), \theta(\xi)), \theta(t)-\theta(\xi)\right] b_{T}(\xi) d \xi$.

and

$$
\hat{p}(t, \mathbf{X})=g(v(t, \mathbf{X}), T) .
$$

This is our principle of time-temperature superposition. It is, of course, an assumption subject to experimental verification.

To phrase our principle in a less precise but more intuitive way, suppose an observer sees a motion at temperature $T_{0}$. Suppose, then, that at temperature $T(t)$ the observer, reading only the temperature sensitive clock, appears to see the same motion again. Then he will appear to see the same history of

$$
\left(\sigma_{i j}+p \delta_{i j}\right) / \rho T .
$$

It is now our job to establish that a perfect elastic fluid is consistent with this principle. To this end, consider the motion $\mathbf{x}=\mathbf{f}(t, \mathbf{X})$ at temperature $T_{0}$. Then

$$
\frac{\sigma_{i j}(t)+p(t) \delta_{i j}}{\rho(t) T_{0}}=\int_{-\infty}^{t} \pi_{i j}\left[x_{k l}(t, \tau), t-\tau\right] d \tau
$$

where

$\pi_{i j}\left[x_{k l}(t, \tau), t-\tau\right]=x_{i k}(t, \tau) x_{j l}(t, \tau) S_{k l}[\mathbf{E}(t, \tau), t-\tau]$

and we have dropped explicit mention of the particle $\mathbf{X}$.

If for the motion $\mathbf{x}(t)=\mathbf{f}(t, \mathbf{X})$ we write for (2.7)

$$
\mathbf{x}(t)=\mathbf{g}[\mathbf{x}(\tau), t, \tau],
$$

we shall get for the motion $\hat{\mathbf{x}}(t)=\mathbf{x}(\theta(t))=\mathbf{f}[\theta(t), \mathbf{X}]$

$$
\begin{aligned}
\mathbf{x}(\theta(t)) & =\mathbf{g}[\mathbf{x}(\theta(\tau)), \theta(t), \theta(\tau)] \\
\hat{\mathbf{x}}(t) & =\mathbf{g}[\hat{\mathbf{x}}(\tau), \theta(t), \theta(\tau)]
\end{aligned}
$$

whence the relative deformation gradients for the two motions, namely $x_{k l}[t, \tau]$ and $\hat{x}_{k l}[t, \tau]$ are related by

$$
\hat{x}_{k l}(t, \tau)=x_{k l}[\theta(t), \theta(\tau)] \text {. }
$$

Now (3.4) and (3.5) yield,

$$
\begin{aligned}
\frac{\hat{\sigma}_{i j}(t)+\hat{p}(t)}{\hat{\rho}(t) T(t)}=\frac{\sigma_{i j}(\theta(t))+p(\theta(t))}{\rho(\theta(t)) T_{0}} \\
\quad=\int_{-\infty}^{\theta(t)} \pi_{i j}\left[x_{k l}(\theta(t), \tau), \theta(t)-\tau\right] d \tau .
\end{aligned}
$$

Making the change of variable from $\tau$ to $\xi$, in (3.8), where

$$
\tau=\theta(\xi), \quad d \tau=b_{T} d \xi
$$

yields
From (3.1)

$$
\theta(t)-\theta(\xi)=\int_{\xi}^{t} b_{T}(\zeta) d \zeta
$$

Putting (3.7) and (3.10) into (3.9) yields

$$
\hat{\sigma}_{i j}=-\hat{p} \delta_{i j}+\hat{\rho} T \int_{-\infty}^{t} \pi_{i j}\left[\hat{x}_{k l}(t, \xi), \int_{\xi}^{t} b_{T}(\zeta) d \zeta\right] b_{T}(\xi) d \xi
$$

which, in view of (3.6), is exactly what (2.14) gives for $\hat{\sigma}_{i j}$. Thus the perfect elastic fluid is consistent with our principle of time-temperature superposition.

\section{The First Law of Thermodynamics}

To write down the first law of thermodynamics it is convenient to distinguish two kinds of derivatives. The material derivative of a quantity $\varphi$ will be written as $\dot{\varphi}$ or $\frac{D \varphi}{D t}$. If we consider $\varphi$ as a function of time and particle label (Lagrangian coordinates), the material derivative of $\varphi$ is defined by

$$
\dot{\varphi}=\frac{D \varphi}{D t} \equiv \frac{\partial}{\partial t} \varphi(t, \mathbf{X}) .
$$

The particle velocity $\mathbf{v}$ is given, for example, by $v_{k}=\dot{x}_{k}$. The spatial derivatives of a quantity $\varphi$ will be written $\varphi_{, k}$. If we consider $\varphi$ as a function of time and position (Eulerian coordinates), the spatial derivatives of $\varphi$ are defined by

$$
\varphi_{, k}=\frac{\partial}{\partial x_{k}} \varphi(t, \mathbf{x})
$$

Conservation of mass, in the form of the equation of continuity, is written

$$
\dot{\rho}+\rho v_{k, k}=0
$$

or, in terms of specific volume

$$
\dot{v}=v v_{k, k}
$$

whence

$$
\rho \dot{v}=v_{k, k}=v_{i, j} \delta_{i j}
$$

We write the first law of thermodynamics, which expresses conservation of energy, in the form

$$
\rho \dot{\epsilon}=\sigma_{i j} v_{i, j}-q_{i, i}
$$

where $\mathbf{q}$ is the heat flux vector. 
Now material differentiation of (2.4) yields, with the aid of (2.5) and (2.6)

$$
\dot{\epsilon}=-p \dot{v}+T \dot{s}+T \dot{\Sigma} .
$$

Differentiation of (2.13) yields

$$
\begin{aligned}
\dot{\Sigma}=S[\mathbf{E}(t, t), 0] b_{T}(t)+\int_{-\infty}^{t} S_{k l} \dot{E}_{k l} b_{T}(\tau) d \tau & \\
& +b_{T}(t) \int_{-\infty}^{t} S_{t} b_{T}(\tau) d \tau .
\end{aligned}
$$

Now from (2.9) and (2.10)

$$
\begin{aligned}
\dot{E}_{k l} & =\frac{1}{2}\left[\frac{\partial v_{i}(t)}{\partial x_{k}(\tau)} \frac{\partial x_{i}(t)}{\partial x_{l}(\tau)}+\frac{\partial x_{i}(t)}{\partial x_{k}(\tau)} \frac{\partial v_{i}(t)}{\partial x_{l}(\tau)}\right] \\
& =\frac{1}{2} v_{i, j}\left[\frac{\partial x_{j}(t)}{\partial x_{k}(\tau)} \frac{\partial x_{i}(t)}{\partial x_{l}(\tau)}+\frac{\partial x_{i}(t)}{\partial x_{k}(\tau)} \frac{\partial x_{j}(t)}{\partial x_{l}(\tau)}\right],
\end{aligned}
$$

where, of course, $v_{i, j}=\partial v_{i}(t) / \partial x_{j}(t)$ may be expressed in terms of $t$ and $\mathbf{x}(t)$ alone.

Noting that, by virtue of (2.18), the first term on the right hand side of (4.4) vanishes, we obtain from (4.3), making use of (4.1), (4.4), and (4.5),

$$
\begin{aligned}
& \rho \dot{\boldsymbol{\epsilon}}=-p v_{i, j} \delta_{i j}+\rho T \dot{s} \\
& +\rho T v_{i, j} \int_{-\infty}^{t} x_{i k}(t, \tau) x_{j l}(t, \tau) S_{k l}\left[\mathbf{E}(t, \tau), \int_{\tau}^{t} b_{T}(\xi) d \xi\right] b_{T}(\tau) d \tau \\
& \quad+\rho T b_{T}(t) \int_{-\infty}^{t} S_{t}\left[\mathbf{E}(t, \tau), \int_{\tau}^{t} b_{T}(\xi) d \xi\right] b_{T}(\tau) d \tau .
\end{aligned}
$$

Using (2.14), we get for (4.6)

$$
\rho \dot{\epsilon}=\sigma_{i j} v_{i, j}+\rho T \dot{s}+\rho T b_{T}(t) \int_{-\infty}^{t} S_{t} b_{T}(\tau) d \tau .
$$

Insertion of (4.7) into the first law of thermodynamics (4.2) yields

$$
\rho T \dot{s}+q_{i, i}=-\rho T b_{T}(t) \int_{-\infty}^{t} S_{t} b_{T}(\tau) d \tau .
$$

In the next section we shall show a very important consequence of this equation.

\section{The Second Law of Thermodynamics}

The law that heat flows against the temperature gradient reads

$$
q_{i} T_{, i} \leqslant 0 .
$$

Coupled with the fact that $b_{T}>0,(2.21)$ and (4.8) imply

$$
\rho T \dot{s}+q_{i, i} \geqslant 0,
$$

whence, using (5.1)

$$
\rho \dot{s}+\frac{q_{i, i}}{T} \geqslant \frac{q_{i} T_{, i}}{T^{2}}
$$

or

$$
\rho \dot{s}+\left(\frac{q_{i}}{T}\right)_{, i} \geqslant 0
$$

which implies that the Clausius-Duhem inequality holds locally as well as globally [5]. Thus with (5.1), the assumption of fading memory tells us that a perfect elastic fluid satisfies the second law of thermodynamics in the Clausius-Duhem form.

Note that we may solve (2.3) for $s$, obtaining, for equilibrium, an expression of $s$ as a function of $v$ and $T$, say

$$
s=h(v, T) .
$$

Similarly, whether or not local thermodynamic equilibrium holds, we may solve (2.5) for $s+\Sigma$, obtaining

$$
s+\Sigma=h(v, T)
$$

or

$$
s=h(v, T)-\Sigma .
$$

Recalling $2^{\circ}$, we see immediately from (5.4) the following: For a given value of $v$ and $\mathrm{T}$, the local entropy $\mathrm{s}$ has its maximum value at local thermodynamic equilibrium. Indeed, for a given configuration and $\mathrm{T}$, the entropy has its maximum value when local thermodynamic equilibrium prevails.

Suppose that from some time $t_{1}$ onward the material is held at constant deformation, and that the temperature is also maintained constant from $t_{1}$ onwards. Then, as can be seen from appendix $A$, the value of local entropy $s$ will tend towards its equilibrium value $h(v, T)$ in a monotonically increasing manner. Further, (5.3) tells us that $s+\Sigma$ remains constant during this process, any decrease in $\Sigma$ being exactly equal to an increase in $s$.

Let us compare the values of local entropy for two cases, one in which local equilibrium prevails $(\Sigma=0)$ and another in which it does not prevail $(\Sigma>0)$. Then if the values of specific volume and temperature are the same in both cases, we shall find that the entropy is less in the latter case than in the former. In particular, at given $v$ and $T$, the presence of shear stresses indicates that the entropy is strictly less than it would be at equilibrium.

Example: For an ideal elastic fluid (2.23), we get for (5.4)

$$
s=C_{V} \ln \frac{C_{V} T}{Y(v)}-\Sigma
$$




\section{Temperature and Energy of Stretching}

Combining (2.4) and (5.3) yields

$$
\epsilon=f(v, h(v, T)) \equiv \alpha(v, T),
$$

where $\alpha$ is appropriately defined. Thus the specific internal energy may be expressed as a function of specific volume and local temperature. This relation holds whether or not local thermodynamic equilibrium prevails.

We define $C_{\Delta}$ by

$$
C_{\Delta} \equiv \frac{\partial \alpha}{\partial T}
$$

so that from (6.1), (6.2), and (4.2) one has

$$
\rho \frac{\partial \alpha}{\partial v} \dot{v}+\rho C_{\Delta} \dot{T}=\sigma_{i j} v_{i, j}-q_{i, i},
$$

which, in view of (4.1), could also be written

$$
{ }_{\rho} C_{\Delta} \dot{T}=\left[\sigma_{i j}-\frac{\partial \alpha}{\partial v} \delta_{i j}\right] v_{i, j}-q_{i, i}
$$

Now suppose that heat is flowing but no deformation is occurring so that the symmetric part of $v_{i, j}$ vanishes, i.e., $v_{i, j}+v_{j, i}=0$. Then (6.4) becomes

$$
\rho C_{\Delta} \dot{T}=-q_{i, i} .
$$

Thus $C_{\Delta}$ is the specific heat capacity at constant deformation and we feel quite justified in assuming $C_{\Delta}>0$.

On the other hand, suppose that the material is adiabatically undergoing isochoric deformation (sometimes called a shearing motion). Then (6.3) becomes

$$
\rho C_{\Delta} \dot{T}=\sigma_{i j} v_{i, j},
$$

whence we deduce that in an adiabatic isochoric motion the temperature is rising or falling depending respectively on whether work is being done on or by the material.

During an adiabatic deformation the specific work done is equal to the change in specific internal energy as implied by (4.2). Thus an upper bound on the specific amount of work which can be taken out of the material adiabatically is given by $\epsilon$ provided that zero is a lower bound on $\epsilon$. Furthermore, when the material is giving up work adiabatically and isochorically, so that $q_{i, i}=0, \dot{v}=0, \sigma_{i j} v_{i, j}<0$, then by (4.3) and (5.2),

$$
\dot{\epsilon} \geqslant T \dot{\Sigma}
$$

which together with (4.2) yields

$$
T \dot{\Sigma} \leqslant v \sigma_{i j} v_{i, j} .
$$

Moreover under these circumstances, as we have just argued, $\dot{T} \leqslant 0$, whence

$$
\frac{D(T \Sigma)}{D t} \leqslant T \dot{\Sigma} \leqslant v \sigma_{i j} v_{i, j}<0 .
$$

From this and $T \Sigma \geqslant 0$ it follows that $T \Sigma$ forms an upper bound on the amount of specific work that may be extracted from the material in recovering adiabatically and isochorically.

The specific Helmholtz free energy, $F$, may be defined by

$$
F \equiv \epsilon-T s .
$$

From (6.1), (5.4), and (6.5), we get

where

$$
F=\Phi(v, T)+T \Sigma
$$

$$
\Phi(v, T) \equiv \alpha(v, T)-T h(v, T) .
$$

It is clear from (6.6) that for given temperature and specific volume, or alternatively for given temperature and configuration, $F$ takes its minimum when local thermodynamic equilibrium prevails. Also, from appendix $A$, we see that for constant configuration and temperature, $F$ will tend monotonically to its equilibrium value.

From (6.5), (4.2), and (5.2)

$$
\begin{aligned}
\rho \dot{F}=\rho \dot{\epsilon}-\rho T \dot{s}-\rho s \dot{T} & =\sigma_{i j} v_{i, j}-\rho s \dot{T} \\
& -\left(\rho T \dot{s}+q_{i, i}\right) \leqslant \sigma_{i j} v_{i, j}-\rho s \dot{T} .
\end{aligned}
$$

Thus, if $\Phi(v, T)$ has a lower bound for each $T$ when $p \geqslant 0$, then the difference between $F$ and this lower bound constitutes an upper bound on the specific amount of work which may be recovered isothermally from the material. In any case, (6.6) and (6.7) tell us that $T \Sigma$ gives an upper bound on the specific amount of work that may be recovered isochorically and isothermally from the material.

If the material is deformed from equilibrium and then allowed to come back to its initial temperature and volume while still in shear, (6.1) tells us that there is no net change in internal energy, and thus, from (4.2), the work done on the material has all been converted into heat, which has been given off to the surroundings. If work is now extracted from the material, and then the material is allowed to return to its original volume and temperature, we similarly see that the material has effectively removed heat from its surroundings and converted it into work. When stresses relax to zero isothermally with no motion of the material, no energy changes or conversions take place. Rather, the material loses its potential to convert heat into work while in contact with isothermal surroundings. The loss of this ability can leave a net increase in the amount of heat that has flowed into the surroundings, giving rise to viscous heat production. Thus, in this theory, elastic behavior and viscous heat production are intimately related in contrast to their frequent introduction as separate phenomena [6]. 
Example: For an ideal elastic fluid, (2.23), we have already obtained the special form of (6.1) namely

$$
\epsilon=C_{V} T \text {. }
$$

Thus for this fluid

$$
C_{\Delta}=C_{V}
$$

and we see that the heat capacity at constant deformation is constant. Indeed, it is a simple exercise to establish that the most general perfect elastic fluid having constant $C_{\Delta}$ is

$$
\epsilon=\Upsilon(v) \exp \frac{1}{C_{V}}[s+\Sigma]+\psi(v)
$$

where $Y>0$ and $\psi$ are functions of $v$ and $C_{V}>0$ is a constant. For (6.10), we also have (6.9). For (2.23) we get from (2.24) and (4.2)

$$
\rho C_{V} \dot{T}=\sigma_{i j} v_{i, j}-q_{i, i},
$$

whence we see that the temperature will be rising or falling when adiabatic work is done on or by the material respectively. And this will hold whether or not volume changes are occurring.

For (2.23), (6.6) becomes

$$
\begin{aligned}
F & =C_{V} T\left[1-\ln \frac{C_{V} T}{Y(v)}\right]+T \Sigma \\
\Phi(v, T) & =C_{V} T\left[1-\ln \frac{C_{V} T}{\Upsilon(v)}\right]
\end{aligned}
$$

and thus, in light of (2.25), $\Phi(v, T)$ has a lower bound for each given $T$ and all $p \geqslant 0$ if and only if $Y(v)$ is bounded away from zero for all values of $v$ for which [n $Y(v)]^{\prime} \leqslant 0$. In particular, $Y(v)=\left[a\left(v-v_{1}\right)^{2}+b\right]^{-1}$, where $a$ and $b$ and $v_{1}$ are positive constants, is so bounded. On the other hand, for an ideal gas

$$
\Upsilon(v)=D v^{1-\gamma}, D>0, \gamma>1,
$$

we have $Y(v) \rightarrow 0$ as $v \rightarrow \infty$, whence $\Phi(v, T)$ has no lower bound for any $T$.

\section{Viscosity-Temperature Relations}

The motion

$$
\begin{aligned}
& x_{1}(t)=x_{1}(\tau)+(t-\tau) \dot{\gamma} x_{2}(\tau) \\
& x_{2}(t)=x_{2}(\tau) \\
& x_{3}(t)=x_{3}(\tau)
\end{aligned}
$$

with $\dot{\gamma}$ constant, represents a simple shear. The relative deformation matrix is

$$
\left\|x_{i k}(t, \tau)\right\|=\left\|\begin{array}{ccc}
1 & (t-\tau) \dot{\gamma} & 0 \\
0 & 1 & 0 \\
0 & 0 & 1
\end{array}\right\|
$$

and

$$
\mathbf{E}(t, \tau)=\frac{1}{2}\left\|\begin{array}{ccc}
0 & (t-\tau) \dot{\gamma} & 0 \\
(t-\tau) \dot{\gamma} & 0 & 0 \\
0 & 0 & 0
\end{array}\right\|
$$

Thus we have, for a simple shear (7.1),

$$
\begin{aligned}
x_{i k}(t, \tau) x_{j l}(t, \tau) S_{k l}[\mathbf{E}(t, \tau) & \left.\int_{\tau}^{t} b_{T}(\xi) d \xi\right] \\
& =\varphi_{i j}\left[(t-\tau) \dot{\gamma}, \int_{\tau}^{t} b_{T}(\xi) d \xi\right]
\end{aligned}
$$

for appropriately defined functions $\varphi_{i j}, i, j=1,2,3$. Thus from (7.2) and (2.14), under isothermal conditions,

$$
\sigma_{12}=\rho T \int_{-\infty}^{t} \varphi_{12}\left[(t-\tau) \dot{\gamma},(t-\tau) b_{T}\right] b_{T} d \tau .
$$

Making the change of variable $\xi=(t-\tau) b_{T}$ in (7.3) yields

$$
\sigma_{12}=\rho T \int_{0}^{\infty} \varphi_{12}\left[a_{T} \dot{\gamma} \xi, \xi\right] d \xi \equiv \rho T H\left(a_{T} \dot{\gamma}\right)
$$

where $a_{T}=1 / b_{T}$ and $H$ is the function defined by (7.4). The viscosity $\eta$ is defined by

$$
\eta=\frac{\sigma_{12}}{\dot{\gamma}}
$$

whence, from (7.4)

$$
\eta=\rho T \frac{H\left(a_{T} \dot{\gamma}\right)}{\dot{\gamma}}
$$

The zero shear viscosity $\eta_{0}$ is defined by

$$
\eta_{0}=\lim _{\dot{\gamma} \rightarrow 0} \eta
$$

whence, from (7.5)

$$
\eta_{0}=\rho T a_{T} \lim _{a_{T} \dot{\gamma} \rightarrow 0} \frac{H\left(a_{T} \dot{\gamma}\right)}{a_{T} \dot{\gamma}}=\rho T a_{T} H^{\prime}(0) .
$$

Thus, from (7.5) and (7.6)

$$
\frac{\eta}{\eta_{0}}=\frac{H\left(a_{T} \dot{\gamma}\right)}{a_{T} \dot{\gamma} H^{\prime}(0)},
$$


i.e., $\eta / \eta_{0}$ is a function of $\mathbf{a}_{\mathrm{T}} \dot{\gamma}$ alone. Now from (7.6)

$$
a_{T}=\frac{\eta_{0}}{\rho T H^{\prime}(0)}
$$

whence, eliminating $a_{T}$ in (7.7) yields

$$
\frac{\eta}{\eta_{0}}=\frac{H\left(\eta_{0} \dot{\gamma} / \rho T H^{\prime}(0)\right)}{\eta_{0} \dot{\gamma} / \rho T}=L\left(\frac{\eta_{0} \dot{\gamma}}{\rho T}\right)
$$

where $L$ is an appropriately defined function.

Equation (7.8) is in agreement with experimental data reported by $\mathrm{F}$. Bueche [7].

\section{Infinitesimal Strain}

Let us expand $S$ in powers of

$$
\begin{aligned}
J_{1} & =\operatorname{tr} \mathbf{E}(t, \tau)=E_{k k}(t, \tau)=\frac{1}{2}\left(I_{1}-3\right) \\
J_{2} & =\operatorname{tr} \mathbf{E}^{2}(t, \tau)=E_{k l}(t, \tau) E_{l k}(t, \tau) \\
& =\frac{1}{4}\left(I_{1}-3\right)^{2}+\left(I_{1}-3\right)-\frac{1}{2}\left(I_{2}-3\right) \\
J_{3} & =\operatorname{det} \mathbf{E}(t, \tau)=\frac{1}{8}\left[I_{3}+I_{1}-I_{2}-1\right] .
\end{aligned}
$$

Because $S[0, t]=S_{k l}[\boldsymbol{0}, t]=0$, we get, to second order in $E_{k l}(t, \tau)$,

$$
\begin{aligned}
& S\left[\mathbf{E}(t, \tau), \int_{\tau}^{t} b_{T}(\xi) d \xi\right] \\
& \quad=\frac{1}{2} \varphi\left(\int_{\tau}^{t} b_{T}(\xi) d \xi\right) J_{1}^{2}+\frac{1}{2} \psi\left(\int_{\tau}^{t} b_{T}(\xi) d \xi\right) J_{2},
\end{aligned}
$$

where $\varphi$ and $\psi$ are appropriate functions. Thus

$$
S_{k l}=\varphi t r \mathbf{E} \delta_{k l}+\psi E_{k l}
$$

to first order in $E_{k l}$.

In appendix B, we show that for infinitesimal strain, with small displacement $\mathbf{u}(t)$ from an undeformed configuration, we may take

$$
\begin{aligned}
& x_{i k}(t, \tau)=\delta_{i k}+u_{i, k}(t)-u_{i, k}(\tau) \\
& E_{i j}(t, \tau)=e_{i j}(t)-e_{i j}(\tau),
\end{aligned}
$$

where

$$
e_{i j}=\frac{1}{2}\left[u_{i, j}+u_{i, j}\right] \text {. }
$$

Assume now that the undeformed configuration is in equilibrium at temperature $T$ and ambient pressure $p_{0}$ with specific volume $v_{0}=v\left(p_{0}, T\right)$ and density $\rho_{0}=$ $\rho\left(p_{0}, T\right)$. Standard infinitesimal strain analysis yields

$$
\begin{aligned}
& \rho(t)=\rho_{0}\left[1-e_{k k}(t)\right] \\
& v(t)=v_{0}\left[1+e_{k k}(t)\right] .
\end{aligned}
$$

Using (2.15), let

$$
\kappa\left(p_{0}, T\right) \equiv g_{v}\left(v_{0}, T\right) v_{0},
$$

where

$$
g_{v} \equiv \frac{\partial g}{\partial v} .
$$

Then, to first order in $v$

$g(v, T)=p_{0}+\frac{\kappa\left(p_{0}, T\right)}{v_{0}}\left(v-v_{0}\right)=p_{0}+\kappa\left(p_{0}, T\right) e_{k k}$.

Substituting (8.3), (8.4), (8.5), (8.6), and (8.8) into (2.16), and dropping all terms of order greater than one in $u_{i, j}$ gives

$$
\begin{aligned}
\sigma_{i j}=- & p_{0} \delta_{i j}-\kappa\left(p_{0}, T\right) \delta_{i j} \\
+ & \delta_{i j} T \int_{-\infty}^{t} \varphi\left(\int_{\tau}^{t} b_{T}(\xi) d \xi\right) b_{T}(\tau) d \tau \operatorname{tre}(t) \\
& +T e_{i j}(t) \int_{-\infty}^{t} \psi\left(\int_{\tau}^{t} b_{T}(\xi) d \xi\right) b_{T}(\tau) d \tau \\
& -T \delta_{i j} \int_{-\infty}^{t} \varphi\left(\int_{\tau}^{t} b_{T}(\xi) d \xi\right) t r \mathbf{e}(\tau) b_{T}(\tau) d \tau \\
& -T \int_{-\infty}^{t} \psi\left(\int_{\tau}^{t} b_{T}(\xi) d \xi\right) e_{i j}(\tau) d \tau,
\end{aligned}
$$

where $t r e=e_{k k}$. If we separate the stress and strain into pressure parts, $\operatorname{tr} \sigma=\sigma_{k k}$, tre, and shear parts, $\tilde{\sigma}_{i j}=\sigma_{i j}-\frac{1}{3} \delta_{i j} \operatorname{tr} \sigma, \tilde{e}_{i j}=e_{i j}-\frac{1}{3} \delta_{i j}$ tre, we get

$\pi(t) \equiv \operatorname{tr} \sigma(t)+p_{0}$

$$
\begin{gathered}
=\operatorname{tre}(t)\left[-\kappa\left(p_{0}, T\right)-\int_{-\infty}^{t} K^{\prime}\left(\int_{\tau}^{t} b_{T}(\xi) d \xi\right) b_{T}(\tau) d \tau\right] \\
+T \int_{-\infty}^{t} K^{\prime}\left(\int_{\tau}^{t} b_{T}(\xi) d \xi\right) \operatorname{tre}(\tau) b_{T}(\tau) d \tau
\end{gathered}
$$

where

$$
K(t)=\int_{t}^{\infty}[3 \varphi(\tau)+\psi(\tau)] d \tau
$$

and

$$
\begin{aligned}
\tilde{\sigma}_{i j}(t)=- & T \tilde{e}_{i j} \int_{-\infty}^{t} G^{\prime}\left(\int_{\tau}^{t} b_{T}(\xi) d \xi\right) b_{T}(\tau) d \tau \\
& +T \int_{-\infty}^{t} G^{\prime}\left(\int_{\tau}^{t} b_{T}(\xi) d \xi\right) \tilde{e}_{i j}(\tau) b_{T}(\tau) d \tau,
\end{aligned}
$$

where

$$
G(t)=\int_{t}^{\infty} \psi(\xi) d \xi
$$


In the first integral of (8.11) we make the change of variable

$$
\zeta(t, \tau)=\int_{\tau}^{t} b_{T}(\xi) d \xi
$$

to get

$$
\tilde{\sigma}_{i j}=T G(0) \tilde{e}_{i j}(t)+T \int_{-\infty}^{t} G^{\prime}\left(\int_{\tau}^{t} b_{T}(\xi) d \xi\right) \tilde{e}_{i j}(\tau) b_{T}(\tau) d \tau .
$$

In changing variables, we have used the assumption that $b_{T}(\zeta)$ is bounded away from zero so that $\zeta(t,-\infty)$ is infinite. Then (8.12) follows upon observing that $G(\infty)$ is zero. Similarly we get from (8.10)

$\pi=\left[-\kappa\left(p_{0}, T\right)+T K(0)\right] \operatorname{tr} \mathbf{e}(t)$

$$
+T \int_{-\infty}^{t} K^{\prime}\left(\int_{\tau}^{t} b_{T}(\xi) d \xi\right) \operatorname{tr} \mathbf{e}(\tau) b_{T}(\tau) d \tau .
$$

In particular, for isothermal stress relaxation, in which $e_{i j}(t)=0$ for $t<0, e_{i j}(t)=$ const. for $t>0,(8.12)$ and (8.13) yield

$$
\begin{aligned}
\tilde{\sigma}_{i j} & =T G\left(b_{T} t\right) \tilde{\mathrm{e}}_{i j} \\
\pi & =\left[-\kappa\left(p_{0}, T\right)+T K\left(b_{T} t\right)\right] \operatorname{tre.} .
\end{aligned}
$$

Example: For an ideal elastic fluid (2.25) gives us

$$
p_{0}=-\left[\ln \Upsilon\left(v_{0}\right)\right]^{\prime} C_{V} T
$$

whence $v_{0}$ is a function of $p_{0} / C_{V} T$. Moreover, from (8.7)

$$
\kappa\left(p_{0}, T\right)=-\left[\ln \Upsilon\left(v_{0}\right)\right]^{\prime \prime} C_{V} T v_{0}
$$

Thus we may solve (8.14) for $v_{0}$ and insert the result into $(8.15)$ to obtain an expression for $\kappa\left(p_{0}, T\right)$ of the form

$$
\kappa\left(p_{0}, T\right)=-\beta\left(\frac{p_{0}}{C_{V} T}\right) C_{V} T,
$$

where $\beta$ is an appropriately defined function.

From this we see that the infinitesimal stress-strain relations for an ideal elastic fluid are given when the three functions $G, K, \beta$, and the constant $C_{V}$ are specified. The relation for the pressure component specializes for an ideal elastic fluid from (8.13) to

$$
\begin{aligned}
\pi=\left[\beta\left(\frac{p_{0}}{C_{V} T}\right) C_{V} T\right. & +T K(0)] \operatorname{tre}(t) \\
& +T \int_{-\infty}^{t} K^{\prime}\left(\int_{\tau}^{t} b_{T}(\xi) d \xi\right) \operatorname{tr} \mathbf{e}(\tau) b_{T}(\tau) d \tau,
\end{aligned}
$$

but the relation for the shear components does not simplify from (8.12).

\section{Discussion}

In our presentation of the theory of perfect elastic fluids, we have concentrated on the mathematical consistency of the formulation, and have tried to keep our theory as simple as possible. Without essential changes in the theory, one could set $\Sigma$ equal to a sum of terms, each similar to the right hand side of (2.13), but each involving a different $b_{T}$, or one could let $b_{T}$ depend upon specific volume $v$ as well as temperature. Numerous other modifications are also possible. We feel that further investigation is needed before deciding whether such modifications are necessary to make our theory describe real materials.

At present it appears to us that our simple formulation can describe elastomers in what rheologists call the rubbery and flow regions. The applicability of the present formulation to the glassy region cannot be decided without further investigation.

Finally we should emphasize that a principal feature of this paper is its new approach to non-equilibrium thermodynamics. It is not a theory of perturbation about equilibrium. Nor need there exist an equilibrium path between two given equilibrium states. We feel that the essence of this approach should be applicable to any thermodynamic process.

\section{References}

[1] B. Bernstein, E. A. Kearsley, and L. J. Zapas, A study of stress relaxation with finite strain, Trans. Soc. Rheology VII, 391410 (1963).

[2] L. J. Zapas and T. Craft, Correlation of large longitudinal deformations with different strain histories, Bull. Amer. Phys. Soc., Series II, 9, No. 3, Abstract DA4, p. 235 (1964).

[3] P. W. Bridgman, The thermodynamics of plastic deformation and generalized entropy, Rev. Mod. Phys. 22, 56-63 (1950).

[4] J. D. Ferry, Viscoelastic properties of polymers, ch. 11, pp 201-247 (John Wiley \& Sons, New York and London, 1961).

[5] C. A. Truesdell, The mechanical foundations of elasticity and fluid dynamics, J. Rational Mech. Anal. 1, 125-300 (1952).

[6] S. L. Koh and A. C. Eringen, On the foundations of non-linear thermo-viscoelasticity, Intern. J. of Engineering Sci. 1, 199229 (1963).

[7] F. Bueche, Non-newtonian viscosity of synthetic rubber and its solutions, J. Appl. Phys. 30, 1114 (1959).

\section{Appendix $\bar{A}$}

We are to show here that if $\mathbf{E}(t, \tau)=0$ and $T(t)=T\left(t_{1}\right)$ for all $t, \tau \geqslant t_{1}$, then $\lim _{t \rightarrow \infty} \Sigma(t)=0$ where $\Sigma(t)$ decreases monotonically.

Since $\mathbf{E}(t, \tau)=0$ for $t>t_{1}$, monotonicity follows from (4.4), (2.18), and (2.21). We have yet to show that $\lim _{t \rightarrow \infty} \Sigma=0$.

Let $\delta$ be any positive number. For $t \geqslant t_{1}$ we have

$0 \leqslant \Sigma=\int_{-\infty}^{t_{1}} S\left[\mathbf{E}\left(t_{1}, \tau\right), \int_{\tau}^{t} b_{T}(\xi) d \xi\right] b_{T}(\tau) d \tau$ 
Because of convergence of the improper integral (A.1) for $t=t_{1}$, there exists a $t_{2}<t_{1}$ such that

$$
\int_{-\infty}^{t_{2}} S\left[\mathbf{E}\left(t_{1}, \tau\right), \int_{\tau}^{t} b_{T}(\xi) d \xi\right] b_{T}(\tau) d \tau<\frac{\delta}{2}
$$

and because of (2.21) this must also hold for $t \geqslant t_{1}$.

Digress now to consider a motion involving only a single step deformation at $t=0$, such that $T=T_{0}$. For such a motion $\Sigma$ becomes at zero time just after the step

$$
\int_{-\infty}^{0} S[\mathbf{E}(0, \tau), t-\tau] d \tau
$$

where $\mathbf{E}=\mathbf{E}(0, \tau)$ is constant. In order for this integral to converge with $S_{t} \leqslant 0$, we must have

$$
\lim _{\xi \rightarrow \infty} S[\mathbf{E}, \xi]=0
$$

for each value of $\mathbf{E}$, and also this convergence must be monotonic. Returning now to (A.1) we thus see that for each fixed $\tau, t_{2} \leqslant \tau \leqslant t_{1}$,

$$
S\left[\mathbf{E}\left(t_{1}, \tau\right), \int_{\tau}^{t} b_{T}(\xi) d \xi\right]
$$

tends monotonically to zero as $t \rightarrow \infty$, whence it must do so uniformly in $\tau, t_{2} \leqslant \tau \leqslant t_{1}$. (Dini's theorem.) Hence for large enough $t$

$\int_{t_{2}}^{t_{1}} S\left[\mathbf{E}\left(t_{1}, \tau\right), \int_{\tau}^{t} b_{T}(\xi) d \xi\right] b_{T}(\tau) d \tau<\frac{\delta}{2}$.

Adding (A.2) and (A.3) gives, with the use of (A.1), that for large enough $t$, we have $|\Sigma|<\delta$ whence our result is established.

\section{Appendix B}

We discuss here in a non-rigorous manner the reduction of finite strain quantities to their infinitesimal strain counterparts. We suppose that a particle is displaced from its initial position $X_{i}$ at time $t$ by $u_{i}(t, \mathbf{X})$. Thus the position of the particle $\mathbf{X}$ at time $t$, namely $x_{i}(t)$ is given by

whence

$$
x_{i}(t)=X_{i}+u_{i}(t, \mathbf{X})
$$

$$
x_{i}(t)=x_{i}(\tau)+u_{i}(t, \mathbf{X})-u_{i}(\tau, \mathbf{X}) .
$$

Thus

$$
\begin{aligned}
x_{i k}(t, \tau) & =\delta_{i k}+\frac{\partial X_{l}}{\partial x_{k}(\tau)}\left[\frac{\partial u_{i}(t, \mathbf{X})}{\partial X_{l}}-\frac{\partial u_{i}(\tau, \mathbf{X})}{\partial X_{l}}\right] \\
& =\delta_{i k}+\frac{\partial X_{l}}{\partial x_{k}(\tau)} \frac{\partial x_{m}(t)}{\partial X_{l}}\left[u_{i, m}(t)-u_{i, m}(\tau)\right]
\end{aligned}
$$

where $u_{i, m}(t)=\partial u_{i}(t, \mathbf{X}) / \partial x_{m}(t)$ and $u_{i, m}(\tau)=\partial u_{i}(\tau, \mathbf{X} /$ $\partial x_{m}(t)$.

From (B.1)

$$
\begin{gathered}
\frac{\partial X_{l}}{\partial x_{k}(\tau)}=\delta_{l k}-\frac{\partial u_{l}(t, \mathbf{X})}{\partial x_{k}(\tau)} \\
\frac{\partial x_{m}(t)}{\partial X_{l}}=\delta_{m l}+\frac{\partial u_{m}(t, \mathbf{X})}{\partial X_{l}} .
\end{gathered}
$$

Putting (B.3) and (B.4) into (B.2) and retaining only terms up to second order in the derivatives of displacement yields

$$
x_{i k}(t, \tau)=\delta_{i k}+u_{i, k}(t)-u_{i, k}(\tau)
$$

to first order.

Inserting (B.5) into (2.9-10) and retaining only first order terms in the derivatives of displacement yields

$$
E_{i j}(t, \tau)=e_{i j}(t)-e_{i j}(\tau)
$$

to first order, where $e_{i j}$ is defined by (8.5). 